\title{
Periodic Mesoporous Organosilica Materials Incorporating Various Organic Functional Groups : Synthesis, Structural Characterization, and Morphology
}

M. A. Wahab, Ichiro Imae", Yusuke Kawakami", and Chang-Sik Ha* Supporting Information 

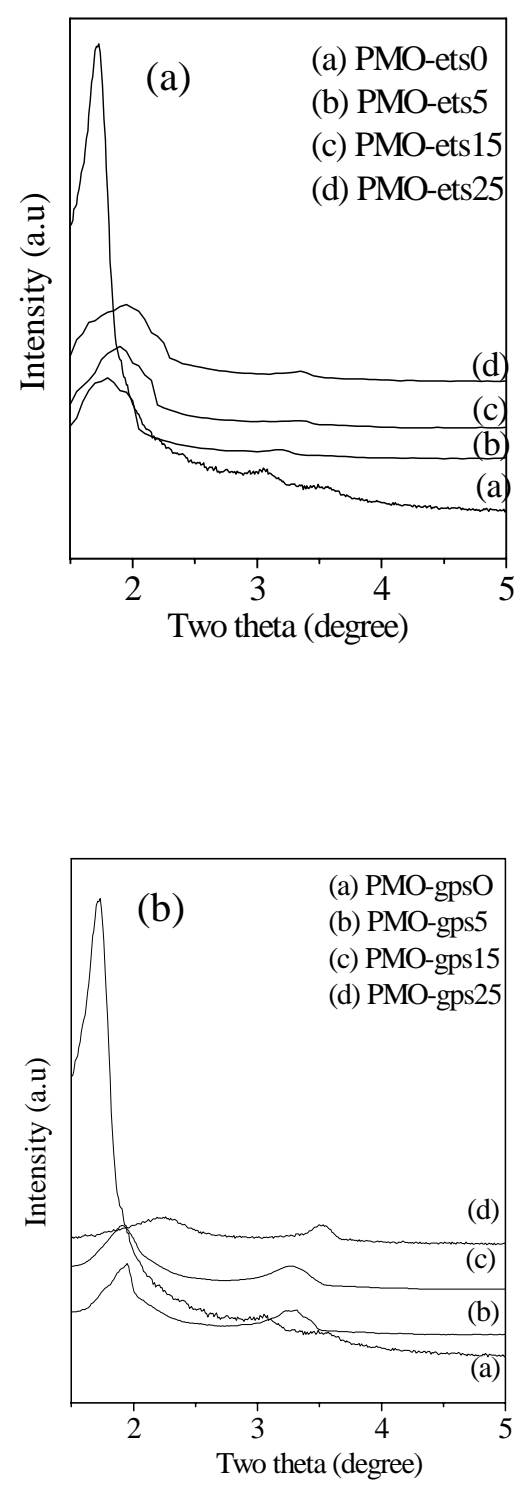

Figure S1. XRD patterns of solvent-extracted (a) PMO-ets series and (b) PMO-gps series. 

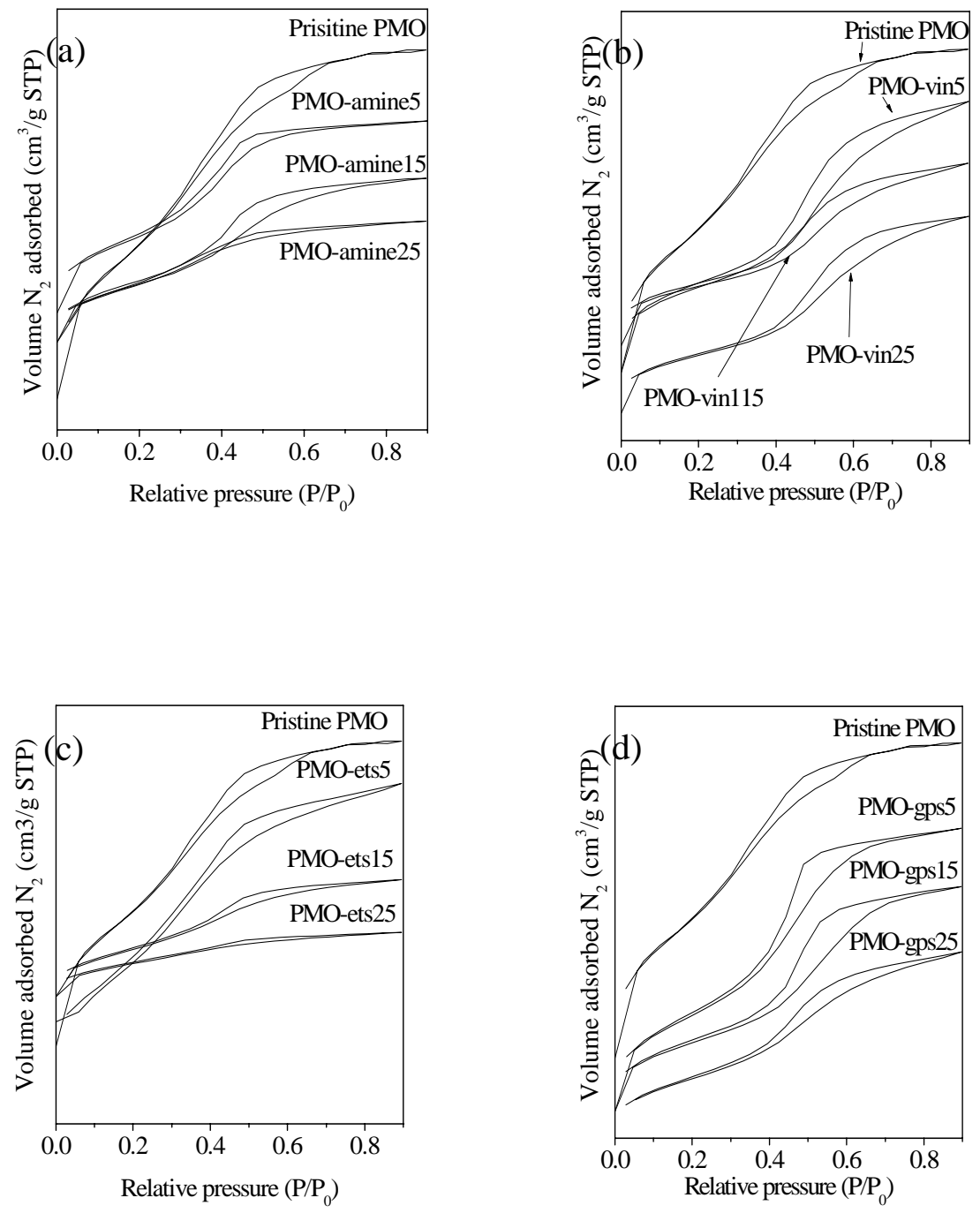

Figure S2. $\mathrm{N}_{2}$ adsorption/desorption isotherms of solvent extracted (a) PMO-amine series, (b) PMO-vin series, (c) PMO-ets series, and (d) PMO-gps series. 
(a)

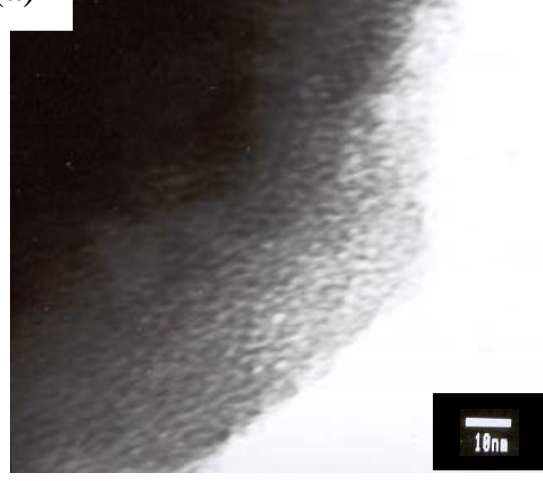

(c)

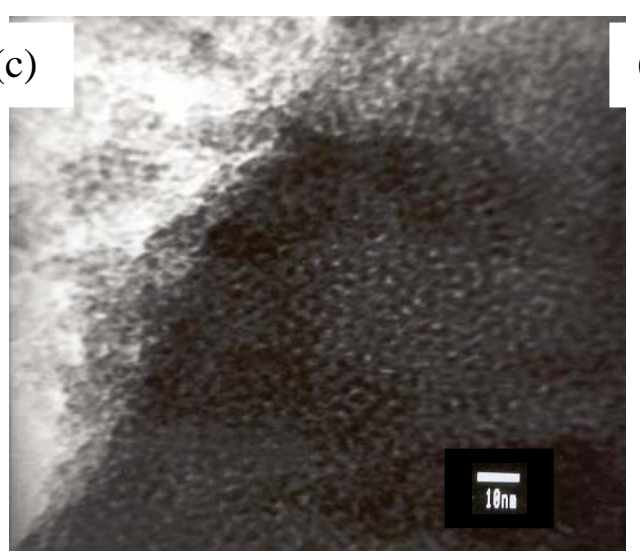

(b)

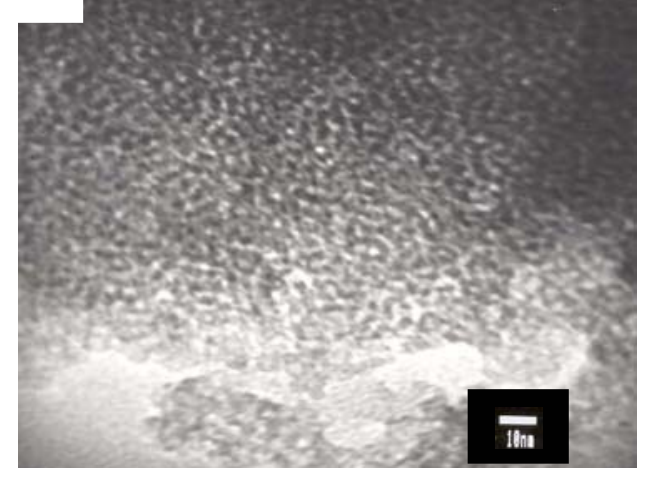

(d)

\section{(e)}
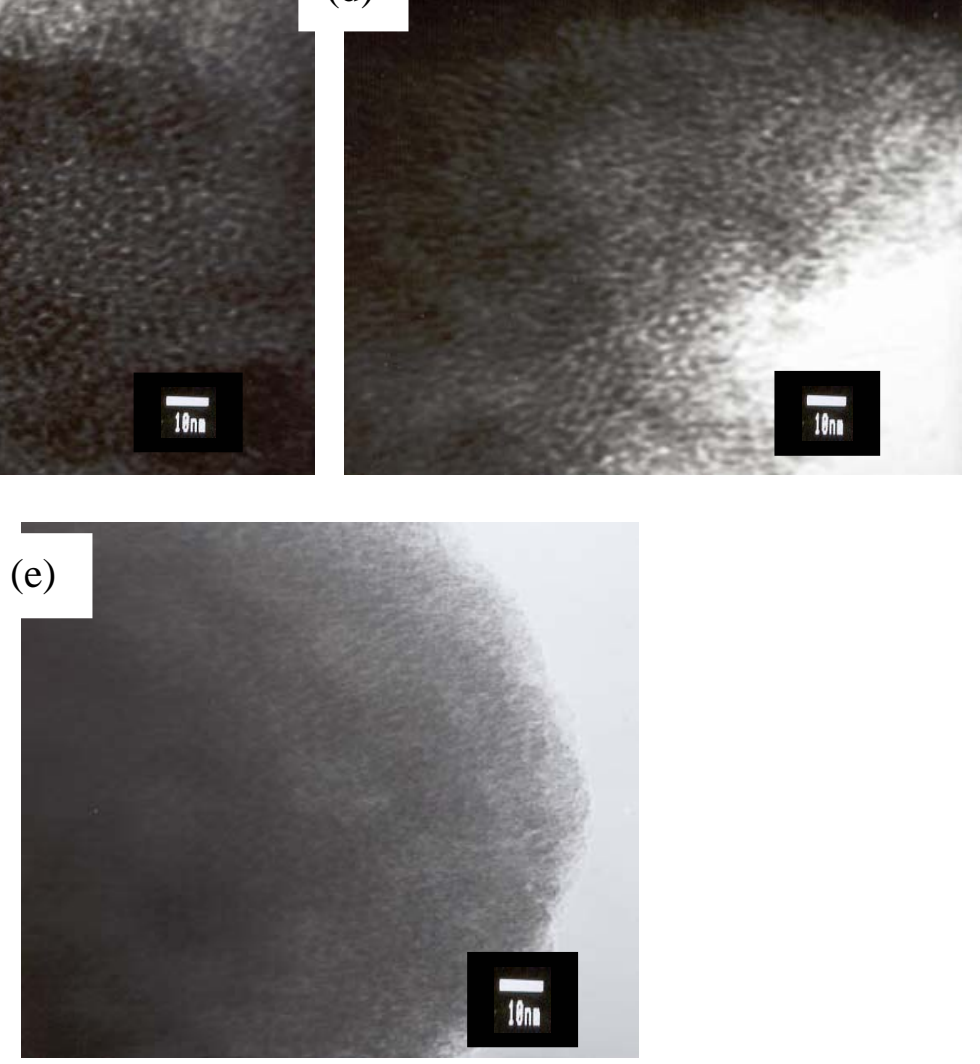

Figure S3. TEM images of solvent extracted functionalized PMOmaterials; (a) PMO-cps25, (b) PMO-amine25, (c) PMO-vin25, (d) PMO-ets25, and (e) PMO-gps25. 

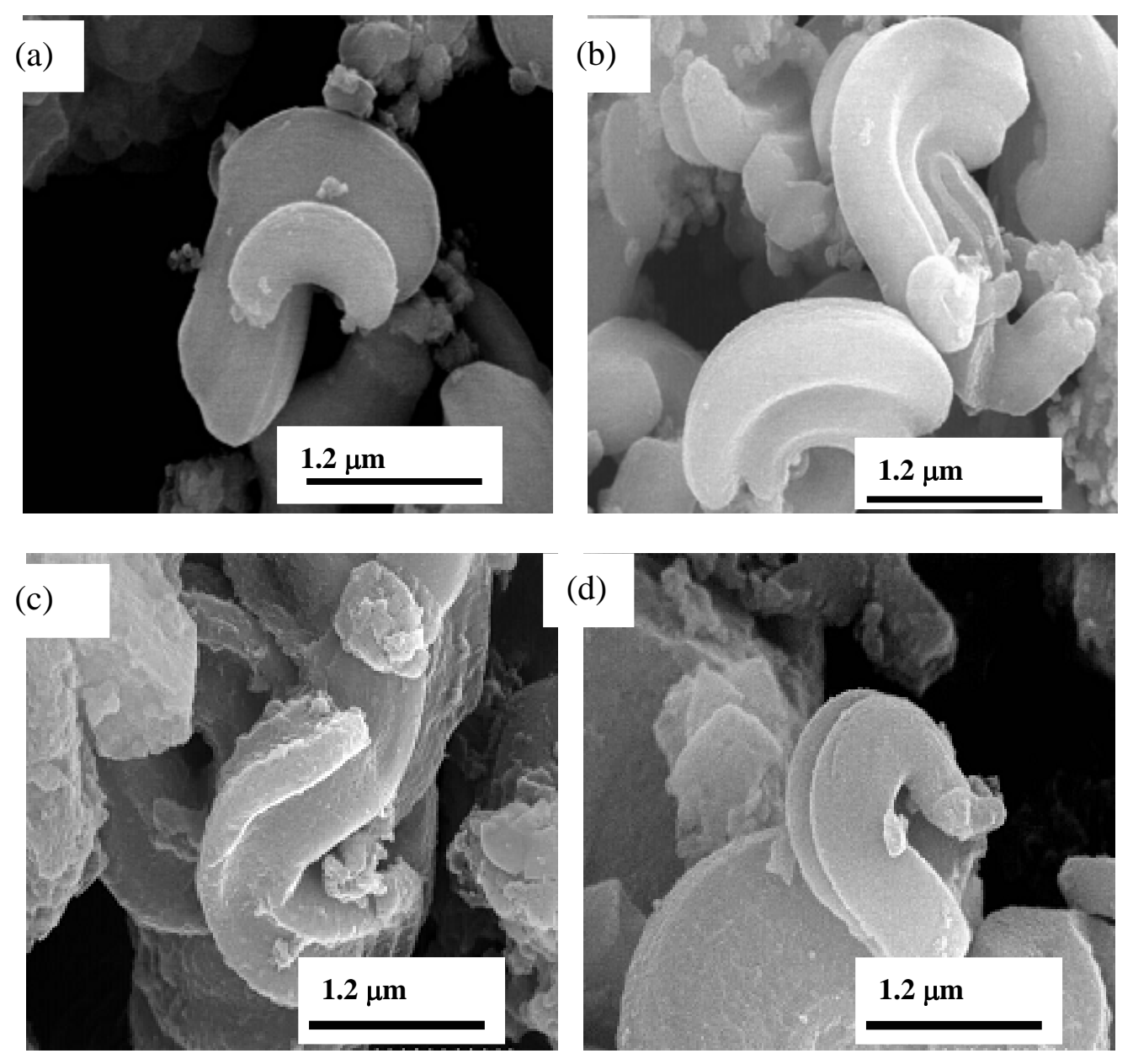

Figure S4. SEM images of (a) PMO-cps5, (b) PMO-cps25, (c) PMO-vin5, (d) PMO-vin25, (e) PMO-amine5, (f) PMO-amine25, (g) PMO-ets5, (h) PMO-ets25, (i) PMO-gps5, and (j) PMO-gps25. (continued) 

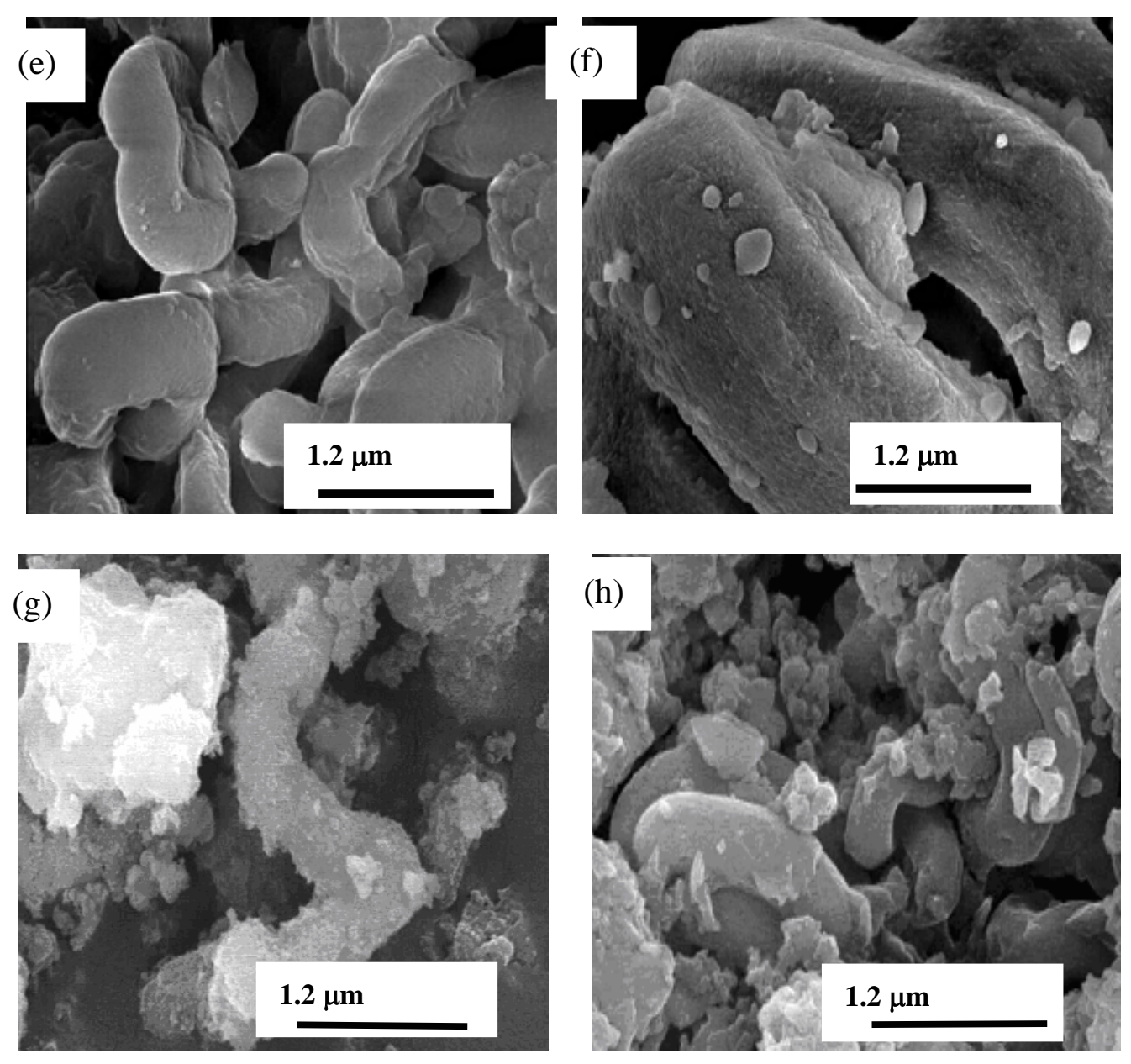

Figure S4. SEM images of (a) PMO-cps5, (b) PMO-cps25, (c) PMO-vin5, (d) PMO-vin25, (e) PMO-amine5, (f) PMO-amine25, (g) PMO-ets5, (h) PMO-ets25, (i) PMO-gps5, and (j) PMO-gps25. (continued) 

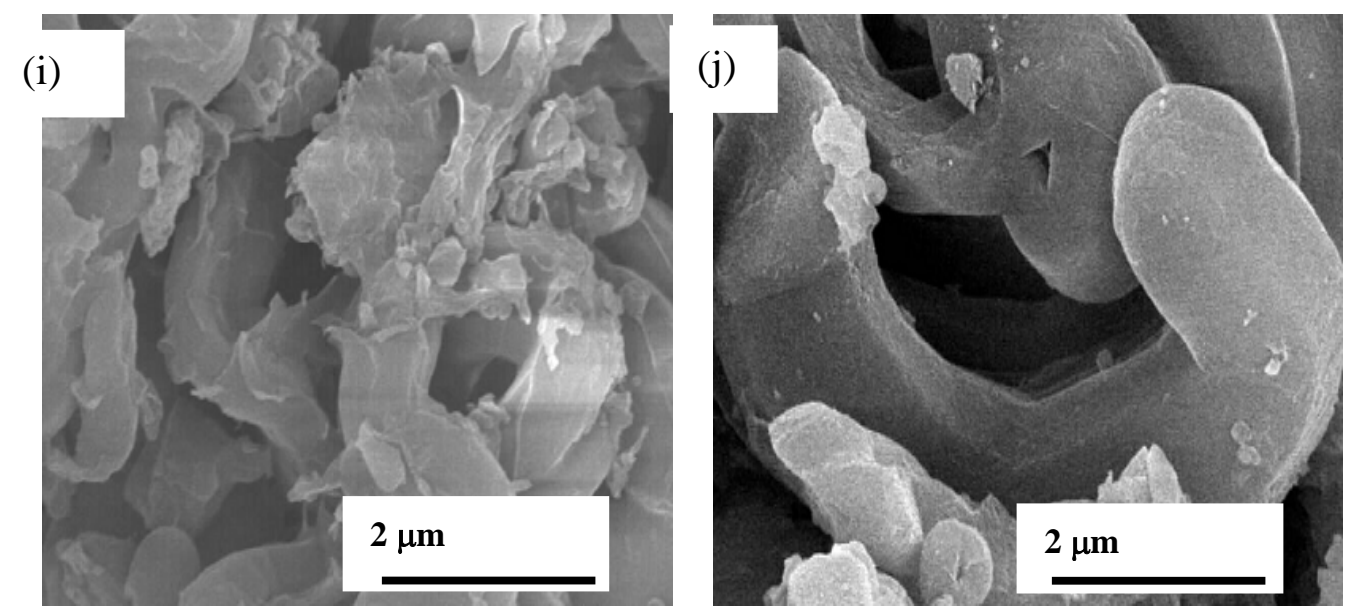

Figure S4. SEM images of (a) PMO-cps5, (b) PMO-cps25, (c) PMO-vin5, (d) PMO-vin25, (e) PMO-amine5, (f) PMO-amine25, (g) PMO-ets5, (h) PMO-ets25, (i) PMO-gps5, and (j) PMO-gps25. 